\title{
Protective effect of renal ischemic postconditioning in renal ischemic-reperfusion injury
}

\author{
Ying Tian ${ }^{1 \#}$, Jia Shu $^{2 \#}$, Ruizhen Huang ${ }^{3 \#}$, Xin $\mathrm{Chu}^{4}$, Xuefeng Mei ${ }^{1}$ \\ ${ }^{1}$ Department of Urology Surgery, ${ }^{2}$ Functional Inspection Division, ${ }^{3}$ Department of Cardiovascular, ${ }^{4}$ Nursing Department, Hospital of Chengdu \\ University of Traditional Chinese Medicine, Chengdu 610039, China \\ Contributions: (I) Conception and design: Y Tian, J Shu, RZ Huang, XF Me; (II) Administrative support: RZ Huang, X Chu; (III) Provision of study \\ materials or patients: Y Tian, J Shu, XF Me; (IV) Collection and assembly of data: All authors; (V) Data analysis and interpretation: Y Tian, RZ \\ Huang, X Chu, XF M; (VI) Manuscript writing: All authors; (VII) Final approval of manuscript: All authors. \\ \#These authors contributed equally to this work. \\ Correspondence to: Xuefeng Mei. Hospital of Chengdu University of Traditional Chinese Medicine, No. 39 Shi-Er-Qiao Road, Chengdu 610072, \\ China. Email: meixuefengmxf@163.com.
}

Background: Renal ischemic postconditioning (RIPo) can protect the kidney from renal ischemia/
reperfusion injury (RIRI). However, the underlying molecular mechanisms for RIPo in renal protection
remained elusive. This study aimed to investigate the renoprotective effects of RIPo in an RIR rat model.
Method: The Sprague Dawley (SD) rats were randomly divided into three groups respectively: sham group,
the RIRI group and the RIPo group. The levels of proteinuria, blood urea nitrogen (BUN), creatinine (Cr),
malondialdehyde (MDA), superoxide dismutase (SOD), lactate dehydrogenase (LDH), reactive oxidative
species (ROS), interleukins (IL)-6, IL-1 $\beta$, and IL-18 were measured by ELISA. Apoptotic cells and caspase-3
positive cells were detected by TUNEL assay and immunohistochemistry, respectively. The protein
expressive levels of caspase-3, caspase-9, ATG8, Beclin1, p62, LC3-II, P-P13K, P-AKT and P-mTOR were
detected by western blot. Results: Our results showed that pretreatment with RIPo significantly reduced ischemic pathological and morphological changes. The levels of proteinuria, BUN, and Cr were also significantly reduced by RIPo pretreatment. Besides, ATG8, LC3-II and Beclin-1 were upregulated in the RIPo group, but p62 was downregulated. Moreover, RIPo pretreatment resulted in higher levels of phosphorylated PI3K, Akt, and mTOR. These results showed that RIPo protects the kidneys of rats from IRI with suppressed apoptosis and activated autophagy. Mechanically, the activated PI3K/AKT/mTOR signaling pathway were activated.

Conclusions: Collectively, our data demonstrated that RIPo could suppress Inflammatory response, oxidative stress, apoptosis and induce autophagy as well as activate the PI3K/AKT/mTOR pathway, which may play an important role in renal protection against RIRI.

Keywords: Renal ischemic postconditioning (RIPo); renal ischemia/reperfusion injury (RIRI); inflammatory response; oxidative stress; apoptosis; autophagy; PI3K/AKT/mTOR pathway

Submitted Mar 23, 2020. Accepted for publication Jun 04, 2020.

doi: $10.21037 /$ tau-20-859

View this article at: http://dx.doi.org/10.21037/tau-20-859

\section{Introduction}

Renal ischemia/reperfusion injury (RIRI), a leading cause of acute kidney injury (AKI), is normally experienced during urological surgery and kidney transplantation, is. RIRI is a potentially life-threatening clinical disease and carries high rates of morbidity and mortality for patients $(1,2)$. The pathogenesis of RIRI is elusive, and several factors are involved in it, including renal endothelial dysfunction, tubular epithelial cell injury, and increased production of 
reactive oxygen species (ROS). These factors all contribute to functional and structural deterioration in the kidney (3). Moreover, the mechanisms of RIRI are also multifactorial. As mentioned in the previous study, oxidative stress, inflammation, cell apoptosis, and autophagy are all involved in the regulation of RIRI (4).

Recently, renal ischemic postconditioning (RIPo) has become a hotspot area that has drawn an increasing amount of interest for its use protecting against ischemia/reperfusion injury (IRI) through "organ conditioning". Interestingly, since the first report by Zhao et al. (5), accumulating evidence has shown that ischemic postconditioning (IPo) can be used to protect a number of different organs (6). Therefore, clinically, IPo has gained recognition as an intervention for protecting organs against RIRI (7). RIPo is a series of transient rapid intermittent ischemia, suitable for reperfusion of previously ischemic kidney (8). RIPo has been explored comprehensively, and studies indicated that RIPo mitigated renal damage after RIRI in rat models (9). We hypothesized that RIPo exhibited a protective role in RIRI.

The phosphoinositide-3-kinase/serine-threonine kinase Akt/mammalian target of rapamycin (PI3K/Akt/mTOR) axis is a well-known pathway that regulates a range of key cellular functions including glucose metabolism, cell proliferation, apoptosis, survival and protein synthesis. An increasing number of studies have suggested that the kidneys and heart can be protected from RIRI via the activation of PI3K/Akt/mTOR signaling $(10,11)$. The PI3K/Akt pathway has been found to negatively regulate genes that facilitate inflammation, thrombosis, and vascular permeability, thus protecting vascular function (12). Activated Akt can rapidly inactivate mTOR and promote autophagy. It is worth noting that the activation of autophagy attenuates RIRI in rats according to the previous studies (2). Given the renal protective role of the PI3K/Akt pathway, it is reasonable to speculate that RIPo may activate the PI3K/Akt/mTOR pathway after RIRI. However, the exact regulatory mechanisms of RIPo are still elusive. The present study was designed to determine whether RIPo attenuates cellular apoptosis, renal lipid peroxidation, and inflammatory responses to reduce RIRI in the kidneys of rats through the activation of autophagy via the PI3K/Akt/ mTOR pathway.

We present the following article in accordance with the ARRIVE reporting checklist (available at http://dx. doi. org/10. 21037/tau-20-859).

\section{Methods}

\section{Animal preparation and experimental protocol}

The Ethics Committee of Experimental Animal Management and Animal Welfare of Chengdu University of Traditional Chinese Medicine reviewed and approved. Code: CD. No20181121c0600130[344].

Six-to-eight-week-old male Sprague Dawley rats (weight: $250-280 \mathrm{~g}$ ) were obtained from the Hubei Laboratory Animal Center (Hubei, China). All animal experiments were carried out in accordance with the National Institute of Health's Guidelines for the Care and Use of Laboratory Animals. The rats were kept in pathogen-free conditions under a $12 \mathrm{~h}$ light/12 h darkness cycle at $25 \pm 3{ }^{\circ} \mathrm{C}$, humidity $60 \%$. They had free access to sterilized water and were fed a standard laboratory diet ad libitum. Animals were observed, Animal preparation was performed as previously described (8). The rats were randomly divided into three groups $(\mathrm{n}=12)$ respectively: the sham-operated control group (sham); the RIRI group: the left renal artery was isolated and clamped for 45 min using a nontraumatic artery clamp after a right nephrectomy where after reperfusion; and the RIPo group: rats were subjected to RIPo prior to I/R, which consisted of 6 cycles of clamping the left renal artery for 10 seconds of reperfusion after that 10 seconds ischemia immediately after 45 minutes of ischemia. The rats were sacrificed 24 hours later. The sham-operated control rats underwent the same process without ligation of the artery.

\section{Proteinuria, blood urea nitrogen (BUN), and creatinine (Cr) determinations}

A murine microalbuminuria ELISA kit, AlbuwellM ${ }^{\mathrm{TM}}$ (Exocell Inc., Philadelphia, PA, USA) was used to detect proteinuria levels. BUN was tested using the QuantiChrom ${ }^{\mathrm{TM}}$ Urea Assay Kit (DIUR-500) (BioAssay Systems, Hayward, CA, USA) in line with the manufacturer's instructions. Serum Cr was measured using the (Enzymatic) Reagent Set from Pointe Scientific, Inc. (Canton, MI, USA).

\section{Assessment of renal injury}

Hematoxylin and eosin staining (H\&E staining) was carried out to assess renal injury histologically using a light microscope. Briefly, the kidney was fixed in $4 \%$ 
paraformaldehyde for $24 \mathrm{~h}$, embedded in paraffin, and sectioned to a thickness of approximately $4 \mu \mathrm{m}$. H\&E staining was performed, and histopathological morphology was observed under a light microscope.

\section{TUNEL assay}

Terminal deoxynucleotidyl transferase-mediated dUTPbiotin nick end labeling (TUNEL) staining was carried out to measure cell apoptosis in situ in accordance with the instructions of the manufacturer (TUNEL Apoptosis detection kit: UPSTATE, Lake Placid, NY, USA). TUNEL-positive cells stained yellowish-brown within the nucleus are apoptotic cells. These cells were observed and counted under the microscope (13). A special OLYMPUS DX51 fluorescence microscope (Tokyo, Japan) was employed to capture images.

\section{Immunobistochemistry}

The renal was fixed with $4 \%$ paraformaldehyde for $24 \mathrm{~h}$, embedded in paraffin, and sectioned. The paraffinized sections were separated with xylene before being rehydrated with gradient ethanol. After the antigen was extracted in $10 \mathrm{mM}$ citric acid buffer, the tissue sections were incubated in $3 \% \mathrm{H}_{2} \mathrm{O}_{2}$ for $10 \mathrm{~min}$ and sealed at room temperature for $1 \mathrm{~h}$. The heart tissue sections were then incubated overnight with a rabbit anti-caspase- 3 antibody (Cell Signaling Technology, Boston, MA, USA). The corresponding second antibody was incubated at room temperature for $1 \mathrm{~h}$. The images were observed under an Olympus DX51 fluorescence microscope (Olympus, Tokyo, Japan). The data were analyzed by Image pro plus 6.0 (Media Cybernetics, USA).

\section{Measurement of oxidative stress markers}

The renal of each rat was removed and homogenized

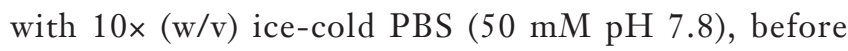
centrifugation at $1,000 \mathrm{rpm} 4{ }^{\circ} \mathrm{C}$ for $3 \mathrm{~min}$. The supernatant was then divided into two: one portion was used to measure lipid peroxidation end product malondialdehyde (MDA) and reactive oxidative species (ROS); and the other underwent centrifugation a second time at $12,000 \mathrm{rpm}$ at $4{ }^{\circ} \mathrm{C}$ for $15 \mathrm{~min}$ and was used to measure superoxide dismutase (SOD) and lactate dehydrogenase (LDH). MDA, ROS, SOD, and LDH were measured with commercially available MDA kits, ROS kits, SOD assay kit, and LDH assay kit (Jiancheng Bioengineering Institute, Nanjing, China), respectively. MDA concentration is expressed as $\mathrm{mmol} / \mathrm{mL}$ of protein, ROS concentration is expressed as $\mathrm{U} / \mathrm{mL}$ of protein, SOD concentration is expressed as $\mathrm{U} / \mathrm{mL}$ of protein, and LDH concentration is expressed as $\mathrm{U} / \mathrm{L}$ of protein.

\section{ELISA}

Commercially available interleukins (IL)-6, IL-1 $\beta$, and IL18 ELISA kits (BD Biosciences, USA) were used to detect inflammatory cytokines, in line with the instructions of the manufacturer. Absorbance was immediately measured using a microplate reader at $450 \mathrm{~nm}$.

\section{Western blotting}

3-methyladenine (3-MA; Selleckchem) was dissolved in $5 \%$ ethanol and intraperitoneally administered at a dose of $300 \mu \mathrm{g} / \mathrm{kg} 20 \mathrm{~min}$ before the onset of ischemia. The rats were sacrificed at $24 \mathrm{~h}$ after RIRI and their kidneys were homogenized as described previously. Total protein was extracted from the kidneys of each group of rats with $1 \mathrm{~mL}$ ice-cold RIPA buffer containing $2 \mathrm{mM}$ PMSF and cocktail. BCA kit (Beyotime Institute of Biotechnology, Shanghai, China) was employed to determine protein concentration. The total protein sample (quantity: $20 \mu \mathrm{g} / \mu \mathrm{L}$ ) was loaded into $10 \%$ sodium dodecyl sulfate-polyacrylamide gel electrophoresis (SDS-PAGE) loading buffer and subsequently transferred to polyvinylidene difluoride (PVDF) membranes. The PVDF membranes were sealed with $5 \%$ skim milk at $37^{\circ} \mathrm{C}$ for 120 min before incubation with the following primary antibodies at $4{ }^{\circ} \mathrm{C}$ overnight, followed by incubating with HRP-conjugated secondary antibodies. Visualization of the signals was carried out with the enhanced Horseradish Peroxidase (Pierce, Rockford, IL, USA). The density of the bands was determined and analyzed using an automatic digital gel image analysis system Bio-Rad CFX-96 (Bio-Rad, CA, USA).

\section{Statistical analysis}

Statistical analysis was carried out with Graph Pad Prism version 5.0 (Sinarline, NY, USA). Data were presented as means $\pm \mathrm{SD}$ for the indicated analyses. Statistical significance in all statistical tests was represented by a probability value of $\mathrm{P}<0.01$. Western blot was evaluated by Quantity One (Discovery Series, USA) analysis. 
A
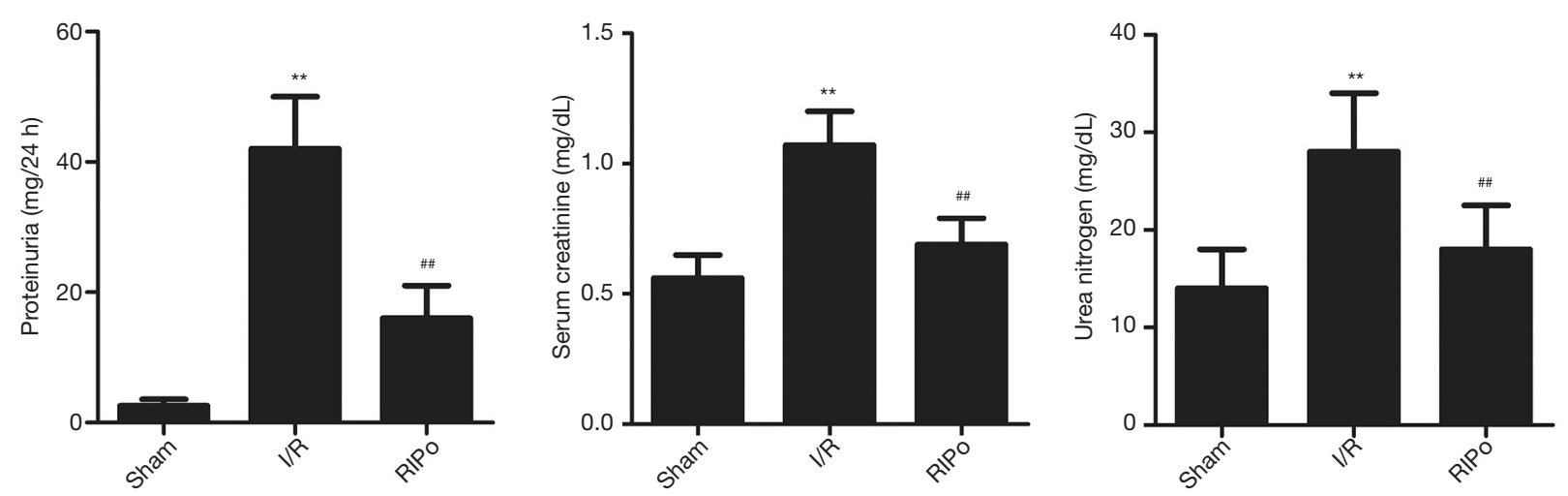

B
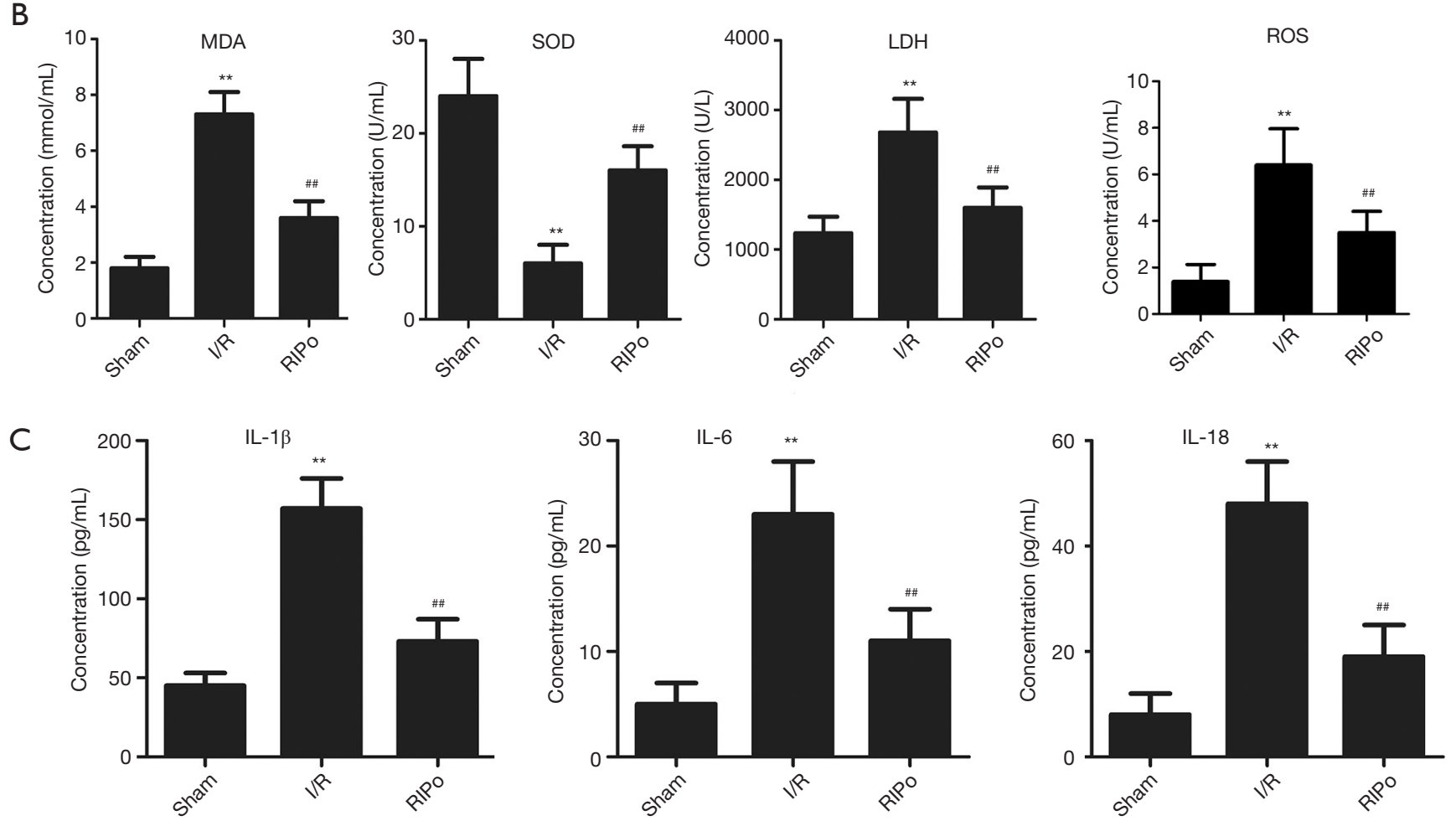

Figure 1 RIPo reduced renal function impairment, oxidative stress level, and inflammation after IRI. (A) Effects of RI-Post on the proteinuria, serum creatinine, and blood urea nitrogen concentrations after 45 minutes of ischemia. (B) MDA, SOD, LDH and ROS concentration at $24 \mathrm{~h}$ post-reperfusion. (C) IL-1 $\beta$, IL-6, and IL-18 concentration in serum at $24 \mathrm{~h}$ post-reperfusion. Bars are means \pm standard error ( $\mathrm{n}=6)$, ${ }^{* *}, \mathrm{P}<0.01$ vs. sham group; ${ }^{\#}, \mathrm{P}<0.01$ vs. I/R group. RIPo, renal ischemic postconditioning; IRI, ischemia/reperfusion injury; RI, renal ischemia; MDA, malondialdehyde; SOD, superoxide dismutase; LDH, lactate dehydrogenase.

\section{Results}

\section{RIPo improves renal dysfunction}

The levels of proteinuria, BUN and Cr were significantly increased in the $\mathrm{I} / \mathrm{R}$ group compared to the sham group. The changes in renal function that were caused by $I / R$ were markedly promoted with RIPo treatment in the RIPo group in comparison to the $\mathrm{I} / \mathrm{R}$ group (Figure $1 A)$.

\section{RIPo decreases oxidative stress and inflammatory cytokines after I/R}

As shown in Figure 1B, MDA and ROS content and SOD and LDH activity in the kidney tissue were measured. 
The MDA content, ROS content and $\mathrm{LDH}$ activity were significantly elevated in the I/R group $(\mathrm{P}<0.01)$, while SOD activity was significantly decreased $(\mathrm{P}<0.01)$. However, the RIPo group showed the reverse results. The serum levels of inflammatory cytokines including IL- $1 \beta$, IL- 6 , and IL18 were detected to determine the extent of pathological inflammation in RIRI. As shown in Figure $1 C$, the levels of IL-1 $\beta$, IL-6, and IL-18 in the serum were significantly increased in the $\mathrm{I} / \mathrm{R}$ group $(\mathrm{P}<0.01)$. However, RIPo significantly decreased serum interleukin levels $(\mathrm{P}<0.01)$.

\section{RIPo improves the morphologic features and attenuates cell apoptosis after RIR}

$\mathrm{I} / \mathrm{R}$ group showed severe pathological changes, as shown in Figure $2 A$, including perivascular dilatation and congestion, tubular dilatation and cellular edema, and protein accumulation in the fluid within the lumen. IPo attenuated the pathological changes. The TUNEL assay showed that the number of TUNEL-positive cells (= apoptotic cells) was significantly elevated in the I/R group in comparison with the sham group. However, TUNEL-positive cells were significantly reduced in number in the RIPo group in comparison to the $\mathrm{I} / \mathrm{R}$ group $(\mathrm{P}<0.01$, Figure $2 B)$. Quantitative analysis of TUNEL-positive cells showed a marked increase in the I/R group, while apoptotic cells were significantly lower $(\mathrm{P}<0.01)$ in the RIPo group than in the I/ $\mathrm{R}$ group (Figure $2 \mathrm{C}$ ). Immunohistochemical staining results revealed that caspase- 3 expression was elevated in the I/R group. Reversely, the expression of caspase- 3 was inhibited in the RIPo group compared to the I/R group (Figure 2D). Quantitative analysis of positive cells for caspase-3 showed an obvious increase in the I/R group, while there was a significant reduction $(\mathrm{P}<0.01)$ in the number of caspase- 3 positive cells in the RIPo group compared with the I/R group (Figure 2E). Western blot results revealed that the protein levels of Caspase- 3 and Caspase- 9 as well as the ratio of $\mathrm{Bcl}-2 / \mathrm{Bax}$ were up-regulated in the I/R group. Reversely, the protein levels of Caspase- 3 and Caspase- 9 as well as the ratio of $\mathrm{Bcl}-2 / \mathrm{Bax}$ were down-regulated in the RIPo group compared to the I/R group (Figure $2 F, G$ ).

\section{RIPo promoting autophagy after I/R}

Expression levels of autophagy-related (ATG) 8, Beclin-1, microtubule-associated protein light chain 3 (LC3)-II, LC3-I, and p62 were analyzed by immunoblotting to elucidate the role of RIPo in autophagy. Western blotting analysis revealed that, compared to the sham group, the levels of ATG8, LC3- II and Beclin-1 were down-regulated in the I/R group, while that of p62 was up-regulated (Figure 3, $\mathrm{P}<0.01$ ). In contrast, I/R-induced autophagy inhibition was alleviated by RIPo. The LC3 II/I ratio was significantly reduced by I/R and restored by RIPo (Figure 3, $\mathrm{P}<0.01)$.

\section{RIPo activated the PI3K/Akt/mTOR pathway}

To investigate whether RIPo can promote autophagy via the PI3K/Akt/mTOR pathway, 3-MA (300 $\mu \mathrm{g} / \mathrm{kg})$, which is known to inhibit autophagy, was administered via intraperitoneal injection. The ratios of $\mathrm{p}-\mathrm{PI} 3 \mathrm{~K} / \mathrm{PI} 3 \mathrm{~K}$, $\mathrm{p}-\mathrm{mTOR} / \mathrm{mTOR}$, and P-Akt/Akt were determined by immunoblotting. Western blot analyses showed that, compared to the sham group, the levels of p-PI3K, p-Akt, and $\mathrm{p}-\mathrm{mTOR}$ were significantly decreased in the I/R group. However, RIPo treatment significantly elevated the expression levels of p-PI3K, p-Akt, and p-mTOR in comparison to those of the $\mathrm{I} / \mathrm{R}$ group (Figure $4 A, \mathrm{P}<0.01$ ). When 3-MA was introduced in the experiment, the levels of p-PI3K, p-Akt, and p-mTOR were significantly lower compared with the RIPo group (Figure $4 A, \mathrm{P}<0.01$ ). The ratios of p-PI3Kp85/PI3Kp85, P-Akt/Akt, and p-mTOR/ mTOR were significantly decreased in RIPo group compared with the sham group. Meanwhile, the ratios of p-PI3Kp85/PI3Kp85 and p-mTOR/mTOR, and p-Akt/ Akt were significantly higher in the RIPo group than the I/R group (Figure $4 B, \mathrm{P}<0.01$ ). However, the ratios of p-PI3Kp85/PI3Kp85 and p-mTOR/mTOR, and p-Akt/ Akt was reversed by adding inhibitor 3-MA compared with RIPo group. Collectively, our data supported that PI3K/ $\mathrm{Akt} / \mathrm{mTOR}$ signaling is activated by RIPo.

\section{Discussion}

RIPo provides a new strategy for protecting the kidney from IRI $(9,14,15)$. The results of the present study are consistent with earlier findings and illuminate the mechanisms of the cellular response to RIRI, which show the therapeutic potential of RIPo. We showed that RIPo ameliorated RIRI in rats by inhibiting cell apoptosis and reducing oxidative stress and inflammation. Interestingly, this protective role against RIRI may result from the activation of the PI3K/ Akt signaling pathway, thus inhibiting the phosphorylation of mTOR.

Given the fact that renal dysfunction, cellular apoptosis, 
A
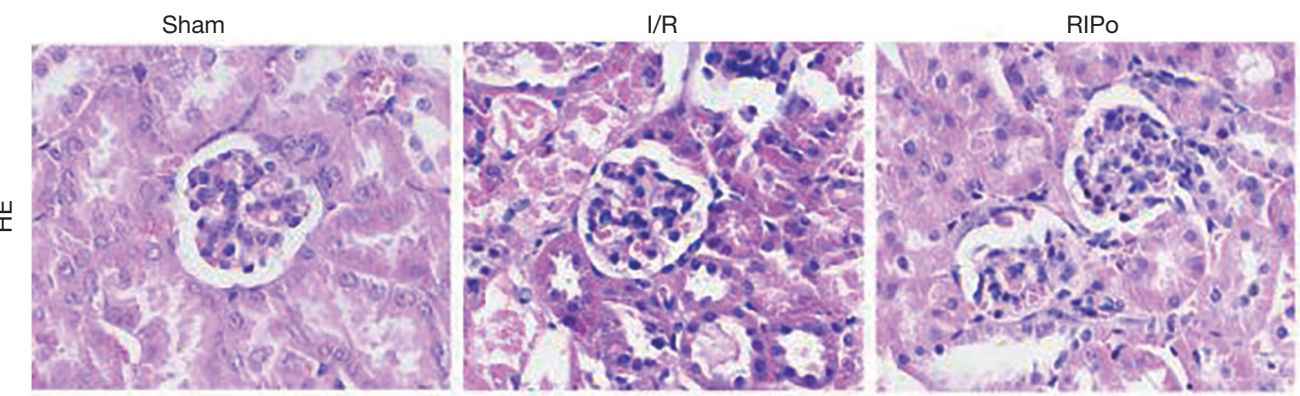

B
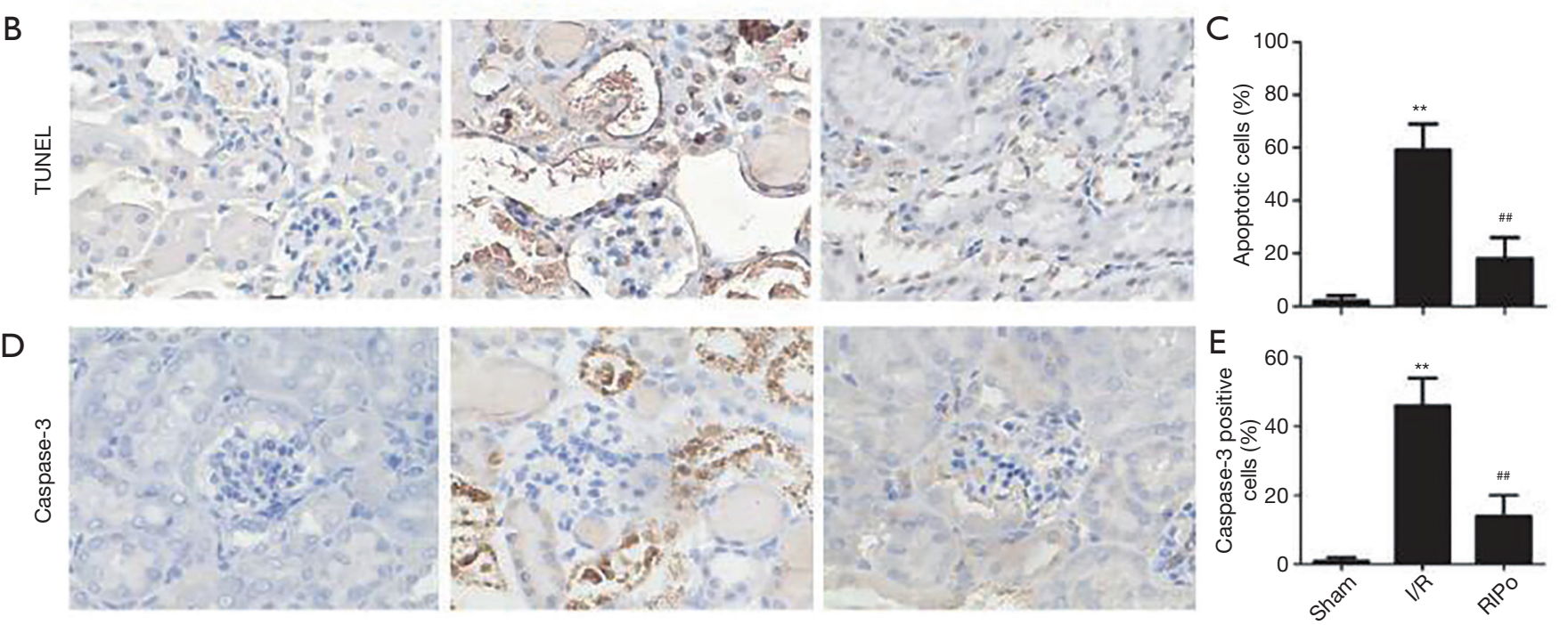

$\mathrm{F}$
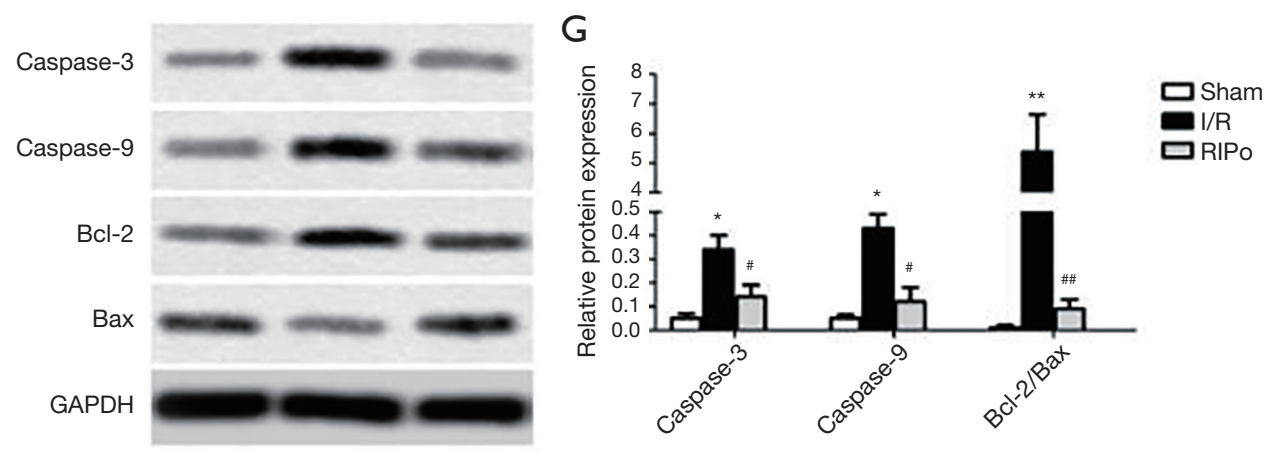

Figure 2 Effect of RIPo on pathological changes and apoptosis after IRI. (A) Representative kidney sections stained with H\&E at the end of 24 hours' reperfusion. (B) Representative photomicrographs of TUNEL staining in the kidney. (C) Immunohistochemistry was performed for the expression of Caspase-3. (D) Percentage of TUNEL-positive cells per high-power field. (E) Percentage of Caspase-3 positive cells per high-power field. Section from a rat treated with ischemic postconditioning. H\&E staining, immunohistochemical staining, and TUNEL assay, original magnification $\times 400$. (F) A representative result for Western blot analysis Caspase-3, Caspase-9, Bcl-2 and Bax. (G) Semi-quantitative analysis of the protein levels of Caspase-3 and Caspase-9 and the ratio of Bcl-2/Bax in each group. Bars are means \pm standard error $(\mathrm{n}=6),{ }^{*}, \mathrm{P}<0.05$ vs. sham group. ${ }^{* *}, \mathrm{P}<0.01$ vs. sham group; ${ }^{*}, \mathrm{P}<0.05$ vs. I/R group. ${ }^{\# \#}, \mathrm{P}<0.01$ vs. I/R group. MDA, malondialdehyde; SOD, superoxide dismutase; LDH, lactate dehydrogenase; IL, interleukins; RIPo, renal ischemic postconditioning; H\&E, hematoxylin and eosin. 


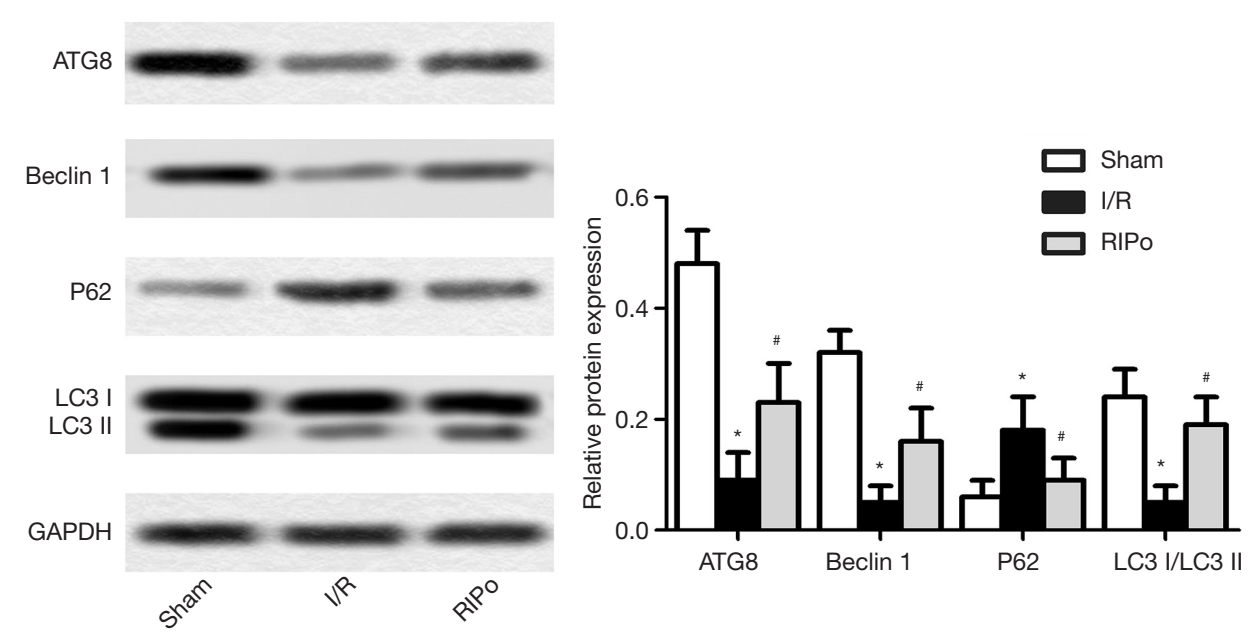

Figure 3 Effect of RIPo on autophagy after IRI. (A) The protein expressions of Beclin-1, LC3, and p62 were determined by Western blotting. (B) Changes in the ratio of LC3-II/LC3-I, P62, and Beclin1protein levels. The GAPDH was used to internal control. Data are presented as the means $\pm \mathrm{SDs}$ in three independent experiments. ${ }^{*}, \mathrm{P}<0.05$ vs. sham group; ${ }^{*}, \mathrm{P}<0.05$ vs. I/R group. I/R, ischemia/ reperfusion; RIPo, renal ischemic postconditioning; RI, ischemia/reperfusion injury.
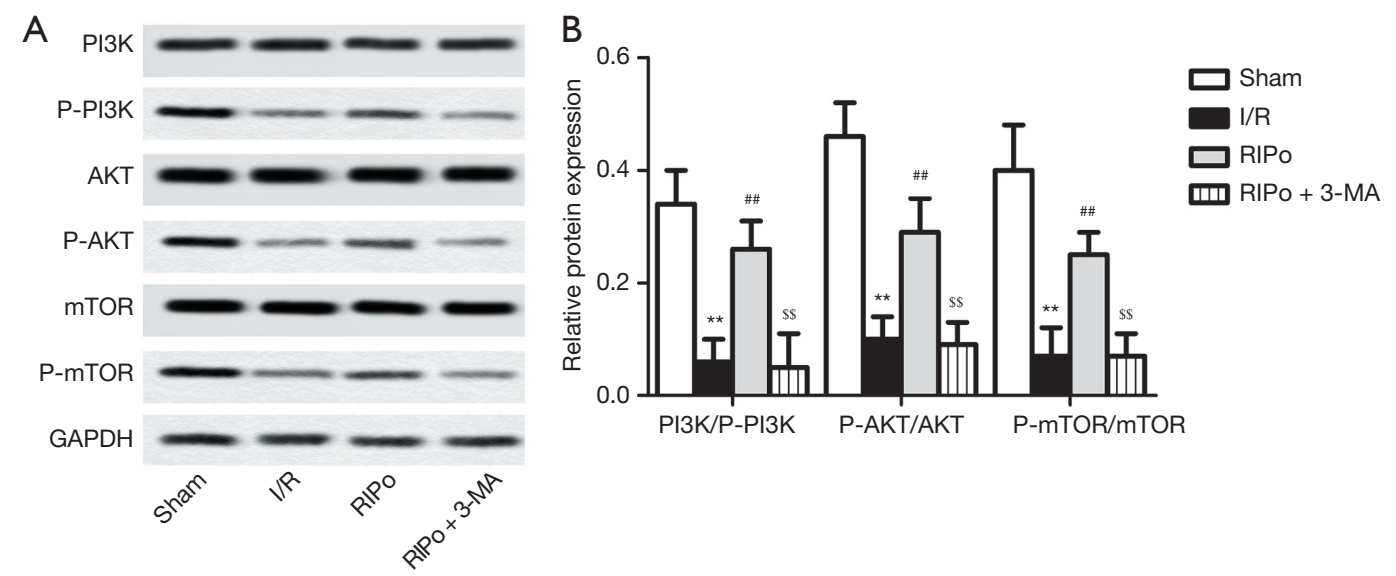

Figure 4 Effect of RIPo on the changes in the phosphorylation of PI3K, Akt, and mTOR after IRI. 3-MA (300 $\mu \mathrm{g} / \mathrm{kg})$ was administered i.p. $20 \mathrm{~min}$ before occlusion of the middle cerebral artery. The protein expressions of p-PI3Kp85, p-mTOR, P-Akt (Ser473), mTOR, PI3K, and Akt were determined by Western blotting. The GAPDH was used to internal control. Data are presented as the means \pm SDs in three independent experiments. **, $\mathrm{P}<0.01$ vs. sham group; ${ }^{\#}, \mathrm{P}<0.01$ vs. I/R group; ${ }^{\text {s }}, \mathrm{P}<0.01$ vs. RIPo group. I/R, ischemia/reperfusion; RIPo, renal ischemic postconditioning; 3-MA, 3-methyladenine; p-PI3K, phospho-PI3K p85.

oxidative stress, and inflammation are involved in RIR, morphological features of renal dysfunction and cellular apoptosis, as well as indexes of renal dysfunction, oxidative stress, and inflammation, were examined. In our study, the I/R group experienced significant acute kidney injury, as shown by severe morphological changes and renal dysfunction. Elevated levels of proteinuria, BUN, and Cr in the I/R group were also observed, suggesting kidney damage had occurred. For our investigation, we subjected rats to 45 min ischemia along with RIPo followed by reperfusion, and assessed RIRI $24 \mathrm{~h}$ after reperfusion. Pretreatment with RIPo was demonstrated to providethe rats with protection against RIRI, as manifested by the restored proteinuria, BUN, and Cr content by RIPo, as well as preserved renal 
function, attenuated pathological changes and inflammation response, reduction of apoptosis, and ROS level.

Apoptosis, also called programmed cell death, is considered a significant contributor to RIRI (16). Caspase-3 and Akt are critical proteins involved in apoptotic pathways. Furthermore, Akt has been reported to be upregulated after IPo, resulting in the inhibition of apoptosis (17). Previous studies have demonstrated that IPo can exert an antiapoptotic effect (18). In our study, Rats subjected to RIPo reduced IRinduced apoptosis, as shown by the significant reduction of TUNELpositive cells (Figure 2B,C). Caspase-3 was seen to be significantly up-regulated after RIRI (Figure 2D,E). However, this up-regulation was significantly decreased after RIPo. Western blotting showed that the anti-apoptotic protein Akt was dominantly expressed after RIPo treatment. Consistent with these studies, our results showed that RIPo significantly inhibited apoptosis, suggesting that RIPo can reduce RIRI via apoptosis inhibition.

Oxidative stress is considered to be a significant factor in IRI-induced damage, which plays a critical role in cell apoptosis. During I/R, the sudden oxygen influx will result in an explosion of ROS in ischemic tissues. It is well known that the MDA content reflects the degree of LPO reactions, while the activities of SOD and $\mathrm{LDH}$ reflect the ability of the body to scavenge ROS. In the RIPo group, we observed decreased levels of MDA, LDH and ROS with increased SOD activity after RIRI. A previous study demonstrated that RIPo significantly reduced RIRI by attenuating renal LPO (17). Consistent with the study, our results showed that RIPo significantly inhibited oxidative stress.

RIRI is a disease positively corrected with inflammatory. In the present study, the ELISA result showed RIPo decreased the serum levels of IL1 $\beta$, IL-6, and IL-18. Previous research reported that IPo attenuated inflammatory response (19). IL1 $\beta$, one of the essential pro-inflammatory cytokines, is generally produced by infiltrated immune cells in injured or infected areas of tissue (20). The previous study revealed that the increase of IL-6 was a marker of inflammatory responses after IRI (21). Similarly, another potent pro-inflammatory cytokine IL-18, also named IFN $\gamma$, has been reported to be involved in ameliorating IRI (22). Consistent with the earlier study, levels of IL1 $\beta$, IL-6, and IL-18 were significantly increased in the rat serum. However, pretreatment with RIPo markedly decreased the serum IL1 $\beta$, IL-6, and IL-18 levels in the rats, showing that RIPo has a protective effect against RIRI in rats via the repression of inflammatory responses.
Autophagy is proved to help cell survival under stress. The yeast ATG8 protein and its mammalian homologues of the LC3 and $\gamma$-aminobutyric acid receptor associated proteins (GABARAP) families have been key for the tremendous expansion of our knowledge about autophagy, a process in which cytoplasmic material become targeted for lysosomal degradation (23). LC3 and Beclin-1 are two pacemakers that contribute to autophagy. A positive correlation has been identified between LC3II/LC3I ratio and the extent of autophagosome formation (24), whereas an inverse correlation has been reported between autophagy and the level of p62 protein (25). IPo has been demonstrated to play a protective role against IRI via the activation of autophagy, both in vitro and in vivo (26). In this study, Beclin-1, LC3II, and the ratio of LC3II/LC3I were increased, but p62 was decreased in the RIPo group, which indicated that RIPo activates autophagy to reduce RIRI. The results agree with the studies above.

The PI3K/Akt pathway plays a part with the regulation of cell proliferation, apoptosis, autophagy, and inflammation (27). mTOR, an essential downstream target of the PI3K/Akt pathway, is vital to protein synthesis and is considered to be a fundamental controller of autophagy (28). A growing bank of evidence has identified that IRI is attenuated via the activation of the $\mathrm{PI} 3 \mathrm{~K} / \mathrm{Akt} / \mathrm{mTOR}$ signaling pathway, which inhibits apoptosis, activates autophagy, and attenuates ROS and inflammatory (29). There is emerging evidence to suggest that mTOR offers protective properties against I/R. For example, RIPo could prevent ischemia-induced apoptosis and memory defects by initiating the mTOR signaling pathway (28). The critical protein expressions of the $\mathrm{PI} 3 \mathrm{~K} / \mathrm{Akt} / \mathrm{mTOR}$ pathway were analyzed to clarify the possible mechanism of the protective response of RIPo against RIR. In our study, reduced levels of PI3K and Akt phosphorylation were seen after RIR compared to the sham group, while enhanced mTOR phosphorylation was also observed. Whereas, RIPo increased the levels of $\mathrm{p}-\mathrm{PI} 3 \mathrm{~K}$ and $\mathrm{p}$-Akt but decreased the level of $\mathrm{p}-\mathrm{mTOR}$. This evidence strongly suggested that PI $3 \mathrm{~K} / \mathrm{Akt} / \mathrm{mTOR}$ pathway plays an essential role in RIR, and RIPo provides protective effects by activating the $\mathrm{PI} 3 \mathrm{~K} / \mathrm{Akt} / \mathrm{mTOR}$ signaling pathway. Herein, these findings are promising, RIPo provides protective effects in the treatment of RIRI, but further experiments are needed to prove whether RIPo plays a role in inhibiting apoptosis and inducing autophagy through the activation of the PI3K/Akt/mTOR signaling pathway. 


\section{Conclusions}

In conclusion, we demonstrated convincing evidence that treatment of RIPo in I/R rats protects RIRI, as manifested by attenuated inflammation and oxidative stress, reduced apoptosis, activated autophagy, and well-preserved renal function. RIPo could suppress Inflammatory response, oxidative stress, apoptosis and induce autophagy as well as activate the $\mathrm{PI} 3 \mathrm{~K} / \mathrm{AKT} / \mathrm{m}$ TOR pathway, which may play an important role in renal protection against RIRI.

\section{Acknowledgments}

The authors thank the lab provided by Chengdu University of Traditional Chinese Medicine.

Funding: None.

\section{Footnote}

Reporting Checklist: The authors have completed the ARRIVE reporting checklist. Available at http://dx.doi. org/10.21037/tau-20-859

Data Sharing Statement: Available at http://dx.doi. org/10.21037/tau-20-859

Conflicts of Interest: All authors have completed the ICMJE uniform disclosure form (available at http://dx. doi. org/10.21037/tau-20-859). The authors have no conflicts of interest to declare.

Ethical Statement: The authors are accountable for all aspects of the work in ensuring that questions related to the accuracy or integrity of any part of the work are appropriately investigated and resolved. The Ethics Committee of Experimental Animal Management and Animal Welfare of Chengdu University of Traditional Chinese Medicine reviewed and approved. Code: CD. No20181121c0600130[344]. All animal experiments were carried out in accordance with the National Institute of Health's Guidelines for the Care and Use of Laboratory Animals.

Open Access Statement: This is an Open Access article distributed in accordance with the Creative Commons Attribution-NonCommercial-NoDerivs 4.0 International License (CC BY-NC-ND 4.0), which permits the noncommercial replication and distribution of the article with the strict proviso that no changes or edits are made and the original work is properly cited (including links to both the formal publication through the relevant DOI and the license). See: https://creativecommons.org/licenses/by-nc-nd/4.0/.

\section{References}

1. Khan OA, Hageman JR, Clardy C. Acute Renal Failure in the Neonate. Pediatr Ann 2015;44:e251-3.

2. Zhang YL, Zhang J, Cui LY, et al. Autophagy activation attenuates renal ischemia-reperfusion injury in rats. Exp Biol Med (Maywood) 2015;240:1590-8.

3. Basile DP, Yoder MC. Renal endothelial dysfunction in acute kidney ischemia reperfusion injury. Cardiovasc Hematol Disord Drug Targets 2014;14:3-14.

4. Eltzschig HK, Eckle T. Ischemia and reperfusion--from mechanism to translation. Nat Med 2011;17:1391-401.

5. Zhao ZQ, Corvera JS, Halkos ME, et al. Inhibition of myocardial injury by ischemic postconditioning during reperfusion: comparison with ischemic preconditioning. Am J Physiol Heart Circ Physiol 2003;285:H579-88.

6. Wever KE, Menting T, Masereeuw R, et al. Local and remote ischemic postconditionings have synergistic protective effects on renal ischemia-reperfusion injury. Transplantation 2012;94:e1-2.

7. Lonborg J, Kelbaek H, Vejlstrup N, et al. Cardioprotective effects of ischemic postconditioning in patients treated with primary percutaneous coronary intervention, evaluated by magnetic resonance. Circ Cardiovasc Interv 2010;3:34-41.

8. Chen H, Xing B, Wang L, et al. Toll-like receptor 4 is involved in renoprotective effect of ischemic postconditioning after renal ischemia/reperfusion injury in rats. Urology 2015;85:483.e1-7.

9. Guo Q, Du X, Zhao Y, et al. Ischemic postconditioning prevents renal ischemia reperfusion injury through the induction of heat shock proteins in rats. Mol Med Rep 2014;10:2875-81.

10. Hu S, Zhang Y, Zhang M, et al. Aloperine protects mice against ischemia reperfusion (IR)-induced renal injury by regulating PI3K/AKT/mTOR signaling and AP-1 activity. Mol Med 2016;21:912-23.

11. Li Q, Shen L, Wang Z, et al. Tanshinone IIA protects against myocardial ischemia reperfusion injury by activating the PI3K/Akt/mTOR signaling pathway. Biomed Pharmacother 2016;84:106-14.

12. Schabbauer G, Tencati M, Pedersen B, et al. PI3KAkt pathway suppresses coagulation and inflammation 
in endotoxemic mice. Arterioscler ThrombVasc Biol 2004;24:1963-9.

13. Wang Q, Cui Y, Lin N, et al. Correlation of cardiomyocyte apoptosis with duration of hypertension, severity of hypertension and caspase-3 expression in hypertensive rats. Exp Ther Med 2019;17:2741-5.

14. Weng X, Shen H, Kuang Y, et al. Ischemic postconditioning inhibits the renal fibrosis induced by ischemia-reperfusion injury in rats. Urology 2012;80:484.e1-7.

15. Khor CS, Wang WJ. The role of acute kidney injury duration in clinical practice. Ann Transl Med 2019;7:S88.

16. Daemen MA, de Vries B, Buurman WA. Apoptosis and inflammation in renal reperfusion injury. Transplantation 2002;73:1693-700.

17. Chen H, Xing B, Liu X, et al. Ischemic postconditioning inhibits apoptosis after renal ischemia/reperfusion injury in rat. Transpl Int 2008;21:364-71.

18. Xia A, Li Y, Li N, et al. Roles of MAPKAPK-2 and HSP27 in the reduction of renal ischemia-reperfusion injury by ischemic postconditioning in rats. Int Urol Nephrol 2014;46:1455-64.

19. Miklos Z, Kurthy M, Degrell P, et al. Ischaemic postconditioning reduces serum and tubular TNF-alpha expression in ischaemic-reperfused kidney in healthy rats. Clin Hemorheol Microcirc 2012;50:167-78.

20. Latz E, Xiao TS, Stutz A. Activation and regulation of the inflammasomes. Nat Rev Immunol 2013;13:397-411.

21. Wang Q, Liu GP, Xue FS, et al. Combined Vagal Stimulation and Limb Remote Ischemic Perconditioning Enhances Cardioprotection via an Anti-inflammatory

Cite this article as: Tian Y, Shu J, Huang R, Chu X, Mei X. Protective effect of renal ischemic postconditioning in renal ischemic-reperfusion injury. Transl Androl Urol 2020;9(3):1356-1365. doi:10.21037/tau-20-85
Pathway. Inflammation 2015;38:1748-60.

22. Liu X, Pan Z, Su D, et al. Remifentanil Ameliorates Liver Ischemia-Reperfusion Injury Through Inhibition of Interleukin-18 Signaling. Transplantation 2015;99:2109-17.

23. Lystad AH, Simonsen A. Mechanisms and Pathophysiological Roles of the ATG8 Conjugation Machinery. Cells 2019;8:973.

24. Mizushima N, Yoshimori T, Levine B. Methods in mammalian autophagy research. Cell 2010;140:313-26.

25. Bjorkoy G, Lamark T, Johansen T. p62/SQSTM1: a missing link between protein aggregates and the autophagy machinery. Autophagy 2006;2:138-9.

26. Qi Z, Dong W, Shi W, et al. Bcl-2 phosphorylation triggers autophagy switch and reduces mitochondrial damage in limb remote ischemic conditioned rats after ischemic stroke. Transl Stroke Res 2015;6:198-206.

27. Nakagawa A, Sullivan KD, Xue D. Caspase-activated phosphoinositide binding by CNT-1 promotes apoptosis by inhibiting the AKT pathway. Nat Struct Mol Biol 2014;21:1082-90.

28. Chen H, Qu Y, Tang B, et al. Role of mammalian target of rapamycin in hypoxic or ischemic brain injury: potential neuroprotection and limitations. Rev Neurosci 2012;23:279-87.

29. Zhang X, He F, Yang J, et al. Protective effects of epigallocatechin-3-gallate on intestinal ischemia reperfusion injury through enhanced activation of $\mathrm{PI} 3 \mathrm{~K} /$ Akt pathway in rats. J Huazhong Univ Sci Technolog Med Sci 2015;35:378-83. 